\title{
ELEMENTAL ABUNDANCES OF SHARP-LINED POP I MAIN SEQUENCE B, A, AND F STARS AND POP II A STARS
}

\author{
SAUL J. ADELMAN ${ }^{1}$, A.G. DAVIS PHILIP ${ }^{2}$ \\ ${ }^{1}$ The Citadel, Department of Physics, Charleston, SC 29409 \\ USA; ${ }^{2}$ Union College, Schenectady, NY 13208 USA; \\ ADELMANS@CITADEL.EDU;AGDP@GAR.UNION.EDU
}

The elemental abundances of late B and early A stars should be among the most reliably derived as these stars have well known atmospheric physics, and non-crowded optical spectra with lines of many species with good oscillator strengths. We obtained elemental abundances of sharp-lined stars from high dispersion, $\mathrm{S} / \mathrm{N}>50$, spectra taken at DAO and KPNO. We performed fine analyses using ATLAS6 and more recently ATLAS9 model atmospheres. Our papers (mostly in MNRAS) contain consistent studies of about 35 B3 to F7 superficially normal, Mercury-Manganese (HgMn), Am, and Field Horizontal Branch (FHB) A stars. The abundances of the normal main sequence $B, A$, and $F$ stars are indicators of Galactic metallicity during the most recent epoch of Galactic history.

The photospheric abundances of FHB A stars, which have not been altered during their stellar evolution, relate to the chemical history of the Galactic Halo. Although the spectrophotometry and spectra of our FHB A stars show them to be the analogues of HB stars in globular clusters with blue horizontal branches, their derived abundances only approximately match those of globular cluster giants. For 8 stars we find that some abundances are correlated, but not those of $\mathrm{Ca}$ and $\mathrm{Fe}$, two standard metallicity indicators. Further the $[\mathrm{Ca} / \mathrm{Fe}]$ values of our Field HB A stars tend to be smaller than those of Population II field giants from which they presumably evolved. Most of the normal B stars have abundances which are slightly less than solar. Understanding this and why the normal early type Population I stars do not always have solar abundances is an important problem. In certain trace species such as $\mathrm{Ga}$, many $\mathrm{Hg}-\mathrm{Mn}$ stars show great overabundances relative to the Sun. These peculiar stars may have an important role in determining the abundances of such elements. 